\title{
Incorporation of Cofilin into Rods Depends on Disulfide Intermolecular Bonds: Implications for Actin Regulation and Neurodegenerative Disease
}

\author{
Barbara W. Bernstein, ${ }^{1,2}$ Alisa E. Shaw, ${ }^{1}$ Laurie S. Minamide, ${ }^{1,2}$ Chi W. Pak, ${ }^{1,2}$ and James R. Bamburg ${ }^{1,2}$ \\ ${ }^{1}$ Department of Biochemistry and Molecular Biology and ${ }^{2}$ The Program in Molecular, Cellular, and Integrative Neuroscience, Colorado State University, \\ Fort Collins, Colorado 80523-1870
}

\begin{abstract}
Rod-shaped aggregates ("rods"), containing equimolar actin and the actin dynamizing protein cofilin, appear in neurons following a wide variety of potentially oxidative stress: simulated microischemia, cofilin overexpression, and exposure to peroxide, excess glutamate, or the dimer/trimer forms of amyloid- $\beta$ peptide $(\mathrm{A} \beta \mathrm{d} / \mathrm{t})$, the most synaptotoxic $\mathrm{A} \beta$ species. These rods are initially reversible and neuroprotective, but if they persist in neurites, the synapses degenerate without neurons dying. Herein we report evidence that rod formation depends on the generation of intermolecular disulfide bonds in cofilin. Of four Cys-to-Ala cofilin mutations expressed in rat E18 hippocampal neurons, only the mutant incapable of forming intermolecular bonds (CC39,147AA) has significantly reduced ability to incorporate into rods. Rod regions show unusually high oxidation levels. Rods, isolated from stressed neurons, contain dithiothreitol-sensitive multimeric forms of cofilin, predominantly dimer. Oligomerization of cofilin in cells represents one more mechanism for regulating the actin dynamizing activity of cofilin and probably underlies synaptic loss.
\end{abstract}

\section{Introduction}

Cofilin is an essential protein with a large range of regulatory mechanisms and functions, including dynamizing actin filament turnover. It can act as a feedback regulator of diverse physiological processes. For that reason any modulation of cofilin activity, including oxidation, is expected to have a profound impact on cellular homeostasis (Bernstein and Bamburg, 2010). For example, oxidation of C39 and C80 to form an intramolecular disulfide bond occurs in T cells following an oxidative burst from granulocytes: it renders cofilin incapable of enhancing actin depolymerization although still able to bind F-actin (Klemke et al., 2008). Under mild oxidative conditions in vitro, cofilin undergoes intermolecular disulfide bonding, not intramolecular, and, when mixed with actin, bundles it into structures (Pfannstiel et al., 2001) that resemble cofilin-actin rods in cells (Minamide et al., 2000, 2010). Here, we present evidence that disulfide bridging of cofilin is needed for the formation of rods in stressed cells.

After their initial reversible generation in many types of cells under stress, rods become stable structures (Bernstein et al., 2006). They are present in Alzheimer disease (AD) brain (Minamide et al., 2000) and are a likely link between a number of

Received Dec. 3, 2011; revised March 2, 2012; accepted March 23, 2012.

Author contributions: B.W.B., A.S., L.M., C.W.P., and J.R.B. designed research; B.W.B., A.S., L.M., and C.W.P. performed research; A.S. contributed unpublished reagents/analytic tools; B.W.B., A.S., C.W.P., and J.R.B. analyzed data; B.W.B. wrote the paper.

This work was supported in part by NIH Grant NS40371, Grant A-4182 from The Cure Huntington Disease Initiative, and a CSU Core Infrastructure support grant for the Microscope Imaging Network.

Correspondence should be addressed to Barbara W. Bernstein, Department of Biochemistry and Molecular Biology, Colorado State University, Campus Delivery 1870, Fort Collins, C0 80523-1870. E-mail: bwb@lamar.colostate.edu.

C.W. Pak's current address is University of Texas Southwestern Medical Center, Dallas, TX.

DOI:10.1523/JNEUROSCI.6020-11.2012

Copyright $\odot 2012$ the authors $\quad 0270-6474 / 12 / 326670-12 \$ 15.00 / 0$ different stresses and the synaptic loss observed in both familial and sporadic $\mathrm{AD}$. The dimer/trimer forms of amyloid- $\beta$ peptide $(\mathrm{A} \beta \mathrm{d} / \mathrm{t})$, the most synaptotoxic forms of $\mathrm{A} \beta$ (Cleary et al., 2005; Mc Donald et al., 2010), also are the most potent rod-inducing forms (Davis et al., 2011). Treatment of cultured neurons with physiologically relevant concentrations $(\sim 250 \mathrm{pM})$ of $\mathrm{A} \beta \mathrm{d} / \mathrm{t}$ increases $\mathrm{A} \beta$ secretion, suggesting a feedforward mechanism appropriate for the accelerating cognitive decline seen in $\mathrm{AD}$. $\mathrm{A} \beta$ induced rods are concentrated in the dentate gyrus region of the hippocampus (Bamburg et al., 2010; Davis et al., 2011), a region with well established importance in learning and memory (Ohm, 2007).

These rods can be immunostained for actin and cofilin/ADF proteins. Mass spectrometry and Western blot analysis of isolated rods confirmed their actin-cofilin composition (Minamide et al., 2010), suggesting a unique structure distinctly different from simple actin aggregation. In fact, electron microscopy shows parallel arrays of filaments (Davis et al., 2009). In the short term, rods protect stressed neurons by slowing loss of mitochondrial potential and neurite ATP (Bernstein and Bamburg, 2003; Bernstein et al., 2006). However, when rods persist, neurites degenerate distal to them (Jang et al., 2005; Minamide et al., 2000). Hence, elucidation of rod dynamics is important for understanding pathology and the regulation of actin assembly in cells.

Glutamate is the major excitatory transmitter in mammalian brain. However, toxic levels of glutamate accumulate when cells are exposed to $\mathrm{A} \beta$ peptide ( $\mathrm{Li}$ et al., 2009) or microischemia, a risk factor for sporadic $\mathrm{AD}$ (Herrup, 2010). Glutamate can stress cells through $\mathrm{Ca}^{2+} / \mathrm{Na}^{+}$overload or by inhibiting cystine uptake, thus depleting glutathione, the major cellular reducing agent (Schubert and Piasecki, 2001). We have focused on cofilin oxida- 

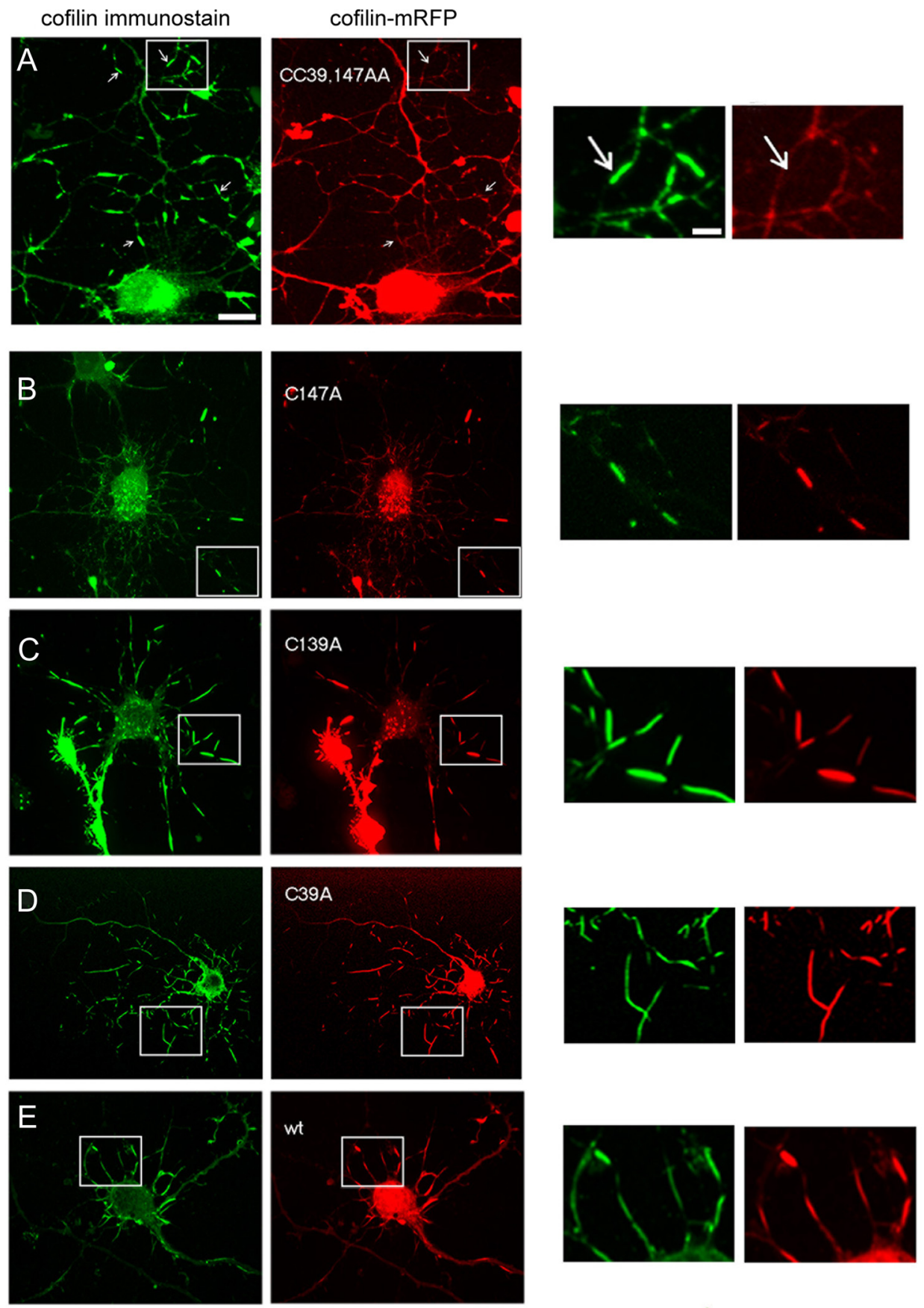

$\mathrm{F}$

(a) inter-molecular
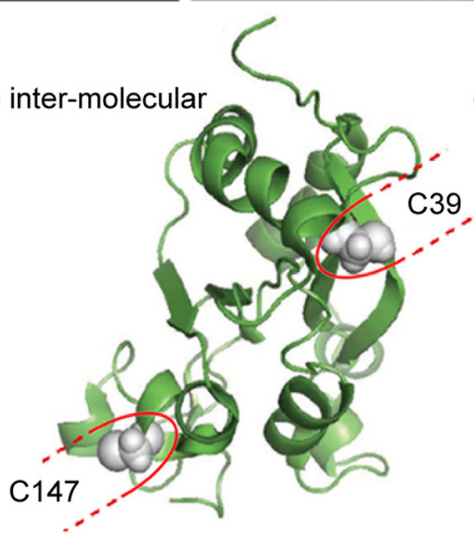

(b) intra-molecular

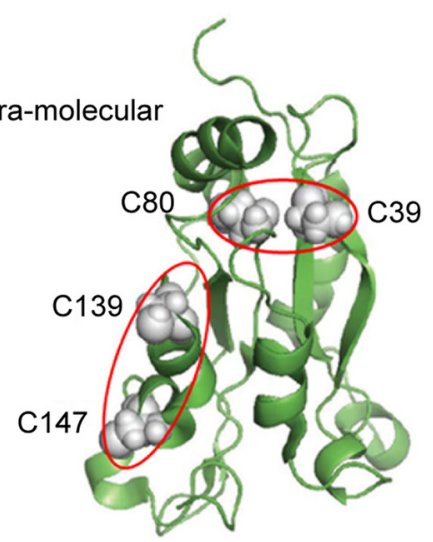

Figure 1. Glutamate-stressed E18 hippocampal neurons, expressing mRFP-cysteine/alanine mutants of cofilin, were immunostained for cofilin. Two small panels on the right in each row are enlargements of boxed regions in left-hand confocal fluorescence images. Arrows in $\boldsymbol{A}$ point to the position of cofilin-actin rods in images of cofilin immunostained and the (Figure legend continues.) 
tion in the glutamate induction of rods in an effort ultimately to understand how environmental factors generate the cytoskeletal pathology typical of both sporadic and familial AD.

\section{Materials and Methods}

All chemical reagents are from Sigma-Aldrich, and all tissue culture and fluorescence reagents are from Life Technologies (Invitrogen) unless otherwise indicated. Cofilin structures are derived from the PyMol program (Schrodinger) using the structural coordinates for cofilin (1Q8) in the Protein Data Bank.

Neuronal cell culture. All mice and rats of either sex were handled according to National Research Council's Guide to the Care and Use of Laboratory Animals as approved by the Institutional Animal Care and Use Committee. Rat E18 cortical and hippocampal neurons were obtained from timed-pregnant dams (Harlan) and were prepared as previously described (Minamide et al., 2000). Cells $(50,000)$ were cultured in $150 \mu \mathrm{l}$ of Neurobasal medium (Invitrogen), supplemented with B-27, GlutaMax $(25 \mu \mathrm{l} / 10 \mathrm{ml})$, and $100 \mu \mathrm{g} / \mathrm{ml}$ penicillin/streptomycin. They were plated on poly-D-lysine-coated no. 1.5, $22 \mathrm{~mm}$ square glass coverslips (Glaswarenfabrik Karl Hecht KG) fixed with high vacuum grease (Dow Corning) to $8 \times 8 \mathrm{~mm}$ glass cloning cylinders (Bellco Glass).

Adenoviral infection and cell treatments. Cultures were maintained in a $5 \% \mathrm{CO}_{2}$ incubator at $37^{\circ} \mathrm{C}$ for $3 \mathrm{~d}$ before infection at $100-200$ multiplicity-of-infection. Experiments were performed $3 \mathrm{~d}$ after infection. Human cofilin constructs [wild-type (wt); C39A; C139A; C147A; CC39,147AA], resistant to human cofilin siRNA silencing, were fused to monomeric Red Fluorescent Protein [mRFP (Campbell et al., 2002)] for virus production as described earlier (Minamide et al., 2003). Transgene expression in hippocampal cells followed Garvalov et al. (2007).

For rod induction, cells were treated for $30 \mathrm{~min}$ at $37^{\circ} \mathrm{C}$ with either $300 \mu \mathrm{M}$ L-glutamate, $10 \mu \mathrm{M} \mathrm{H}_{2} \mathrm{O}_{2}$ in cell culture medium, or $10 \mathrm{~mm} \mathrm{Na}$ azide/ $6 \mathrm{~mm}$ 2-deoxy-D-glucose in PBS to block synthesis of ATP and thus deplete it. In some cases, rods were reversed with $4 \mu \mathrm{M} \beta$-mercaptoethanol (Arcos Organics). For AMPA ( $\alpha$-amino-3-hydroxy-5-methyl-4-isoxazolepropionic acid) experiments, the following glutamate antagonists were added $15 \mathrm{~min}$ before glutamate: DNQX (6,7-dinitroquinoxaline-2-3-dione; $50 \mu \mathrm{M})$, AP5 (DL-2amino-5-phosphonopentanoic acid; $100 \mu \mathrm{M}$ ), and (R,S)-MCPG [S(+)- $\alpha$ Amino-4-carboxy- $\alpha$-methyl-benzeneacetic acid; $500 \mu \mathrm{M}$ ]. In agonist experiments, the agonist alone or combinations of agonists were added: AMPA $(25 \mu \mathrm{M})$; NMDA ( $N$-methyl-D-aspartic acid; $100 \mu \mathrm{M})$; and DHPG [(S)-3,5-dihydroxyphenylglycine; $100 \mu \mathrm{M}]$, a metabotropic glutamate receptor agonist. In other experiments, after cells were stressed, they were incubated either for $30 \mathrm{~min}$ at $37^{\circ} \mathrm{C}$ in $30 \mu \mathrm{M}$ ThiolTracker Violet (Invitrogen Cat. no. T10095) or for $10 \mathrm{~min}$ in $1 \mu \mathrm{M}$ MitoTracker Green FM (Invitrogen Cat. no. M7514), a dye whose uptake is insensitive to mitochondrial membrane potential; they were then washed and fixed. These reagents were stored frozen in DMSO $\left(-20^{\circ} \mathrm{C}\right)$.

For knock down experiments, $3 \mathrm{~d}$ after plating rat hippocampal neurons were infected with AdTrack virus for coexpression, driven by separate promoters, of GFP and of a hairpin RNA to generate a rat cofilin siRNA. In control cells, a hairpin RNA with no target in rat neurons and GFP were coexpressed; this hairpin RNA generates a siRNA targeted to mouse ADF. Three days later the neurons were also infected with virus for expression of wt cofilin-mRFP or CC39,147AA cofilin-mRFP. Two days after the last infection, GFP-positive neurons were scored for cofilinmRFP rods.

Immunocytochemistry. Cells were fixed in $0.1 \%$ glutaraldehyde for 10 min at room temperature, permeabilized with methanol (chilled at

(Figure legend continued.) absence of CC39,147AA-mRFP in those same rods in mRFP images. In $\boldsymbol{B}-\boldsymbol{D}$, single mutants fused to $\mathrm{mRFP}$ show similar high concentrations in rod regions as do immunostained cofilin and wt-mRFP (E). Only the C(39,147AA-mRFP mutant does not accumulate in the regions of immunostained rods. Scale bars: (in large panel of $A$ ) all large panels $\boldsymbol{A}-\boldsymbol{E}, 10 \mu \mathrm{m}$; (in small panel of $\boldsymbol{A}$ ) all small panels $\boldsymbol{A}-\boldsymbol{E}, 5 \mu \mathrm{m}$. $\boldsymbol{F}$, In molecular models red semiellipses in $\boldsymbol{a}$ indicate residues that engage in intermolecular disulfide bonds (Pfannstiel et al., 2001) and in $\boldsymbol{b}$ the ellipses indicate the two most likely intramolecular bonds (Klamt et al., 2009; Klemke et al., 2008). $\left.-20^{\circ} \mathrm{C}\right)$ and $\mathrm{NaBH}_{4}(10 \mathrm{mg} / \mathrm{ml})$ for $3 \mathrm{~min}$ and blocked with $2 \%$ goat serum in $1 \%$ bovine serum albumin/ Tris-buffered saline before immunostaining. Primary antibodies were the same as used for immunoblotting: actin (clone C4, MP Biomedicals) and affinity purified rabbit polyclonal $1439(2 \mathrm{ng} / \mu \mathrm{l})$, a pan antibody for mammalian ADF and cofilin-1 (Shaw et al., 2004). Secondary goat anti-rabbit antibodies (1:450 dilution) were labeled with fluorescein, Alexa 648, or Alexa 488. Coverslips were mounted with ProLong Gold Antifade (Invitrogen).

Fluorescence microscopy and image analysis. An Olympus IX81 microscope was used that was equipped with a heated, programmable encoded ASI piezo stage (Applied Scientific Instrumentation), CSU22 spinning disk confocal head (Yokogawa Instruments), diode lasers (440 nm, 660 $\mathrm{nm}$, Cobalt; $473 \mathrm{~nm}, 561 \mathrm{~nm}$, Crystal), xenon lamp, a $1 \mathrm{~K} \times 1 \mathrm{~K}$ Cascade II EMCCD camera (Roper Scientific), and PlanApo/340 40× (1.35 NA), PlanApo N 60× (1.42 NA), and UPlanSApo 100× (1.40 NA) objectives. The imaging system is controlled through SlideBook v.5 (Intelligent Imaging Innovations). All image analysis was done on maximum intensity projections of 3-4 optical slices using MetaMorph v.7.7 (Molecular Devices) with data transferred to Excel 2007 (Microsoft). In all experiments, only cells with similar moderate levels of expressed protein were compared. Expression levels were estimated from the intensity average of the largest square region that could be fit into the soma. Statistical significance was calculated using Student's $t$ test. Asterisks on figures indicate significant difference between treatment groups at the $p<0.01$ confidence level.

Rod isolation, SDS-PAGE, and immunoblotting. Rat E18 cortical neurons and human epithelial A431 cells were ATP depleted (as above described) to generate rods. Rod isolation, SDS-PAGE, and immunoblotting were done as previously described (Minamide et al., 2010) with the following modification. The rod preparations were divided equally into two Microfuge tubes. Iodoacetamide (IAA) in Pipes buffer was added ( $2.5 \mathrm{~mm}$ final) to both tubes before cell lysis and incubated for 10 min at room temperature. SDS (1\% final) in Pipes buffer with dithiothreitol (DTT, $10 \mathrm{~mm}$ ) was added to one tube and without DTT to the other before both were incubated for $30 \mathrm{~min}$ at room temperature. The proteins were then precipitated with chloroform and methanol (Wessel and Flügge, 1984). Pellets were resolubilized in SDS buffer with or without DTT and separated on 15\% isocratic SDS polyacrylamide gels, and Western blots were prepared. The primary antibodies were those used for immunocytochemistry; secondary antibodies conjugated to Dy-Light were used with the Li Cor Biosciences Odyssey scanner to visualize bands.

Cofilin/F-actin sedimentation assay. Chicken actin was purified (Pardee and Spudich, 1982) and assembled (18.7 $\mu \mathrm{M})$ for $2 \mathrm{~h}$ at room temperature in F-actin buffer (mM: $100 \mathrm{KCl} ; 2 \mathrm{MgCl}_{2} ; 0.2 \mathrm{ATP} ; 0.2$ EGTA; 0.5 DTT; 10 Pipes pH 6.8). Human cofilin and mutant forms were expressed in bacteria and purified on a dye matrix Green A column (Giuliano et al., 1988). F-actin $(5 \mu \mathrm{M})$ was incubated in F-actin buffer with increasing amounts of cofilin $(0,2.5,5,10,20 \mu \mathrm{M})$ and centrifuged at $250,000 \times \mathrm{g}, 20^{\circ} \mathrm{C}, 30 \mathrm{~min}$. Under these conditions, cofilin alone did not sediment. Starting material, pellet, and supernatant fractions were separated by standard 15\% isocratic SDS-PAGE. Proteins were detected by Coomassie Blue R gel staining. Gels were imaged with a Star CH 250 CCD camera (Photometrics), and images were quantified by Total Lab 1D gel software (Nonlinear Dynamics).

\section{Results \\ Cofilin incorporation into rods depends on intermolecular disulfide bonds}

To test the importance of cofilin oxidation in the glutamate induction of neuronal rods, we mutated cysteine residues that were suggested by in vitro studies to be involved (Pfannstiel et al., 2001). The single C/A mutants (C39A, C139A, C147A), double mutant (CC39,147AA), and wt cofilin were fused to mRFP and expressed in rat E18 hippocampal neurons via adenoviral infection. These are hereafter referred to as C39A-mRFP, C139AmRFP, C147A-mRFP, CC39,147AA-mRFP, and wt-mRFP. Figure $1 A$ shows a representative sample of images from eight experiments, performed over several months, that consistently 


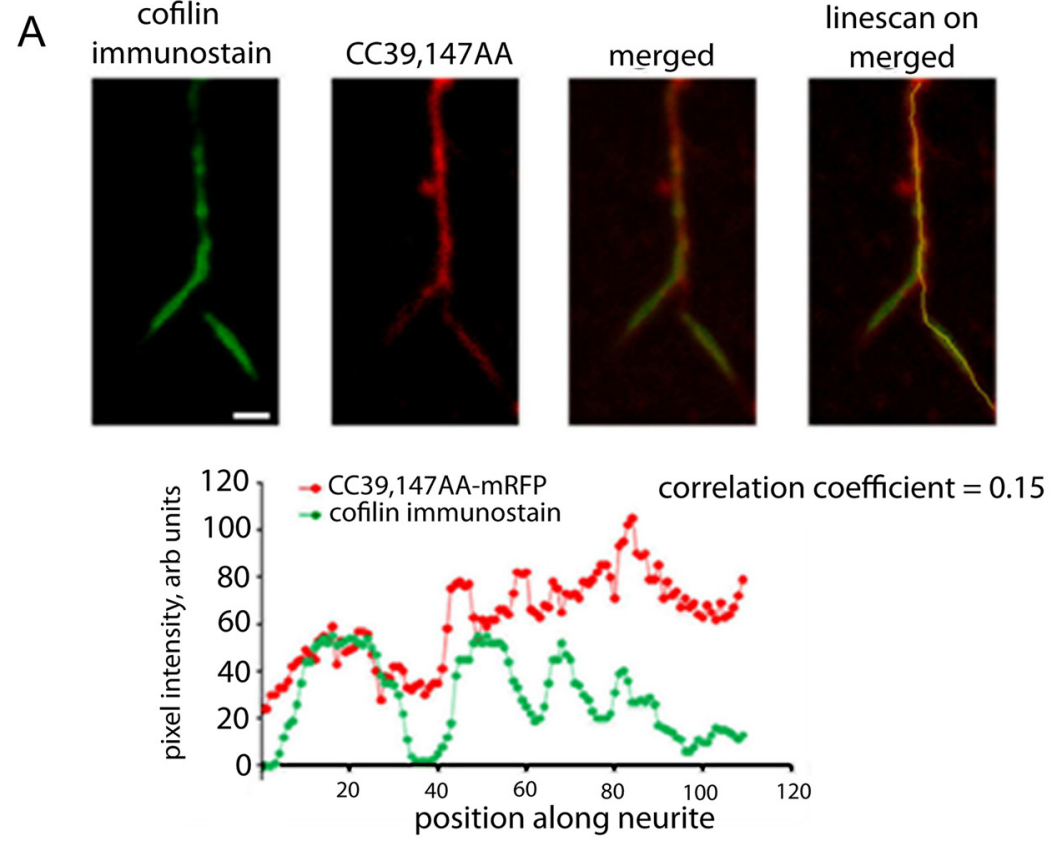

B
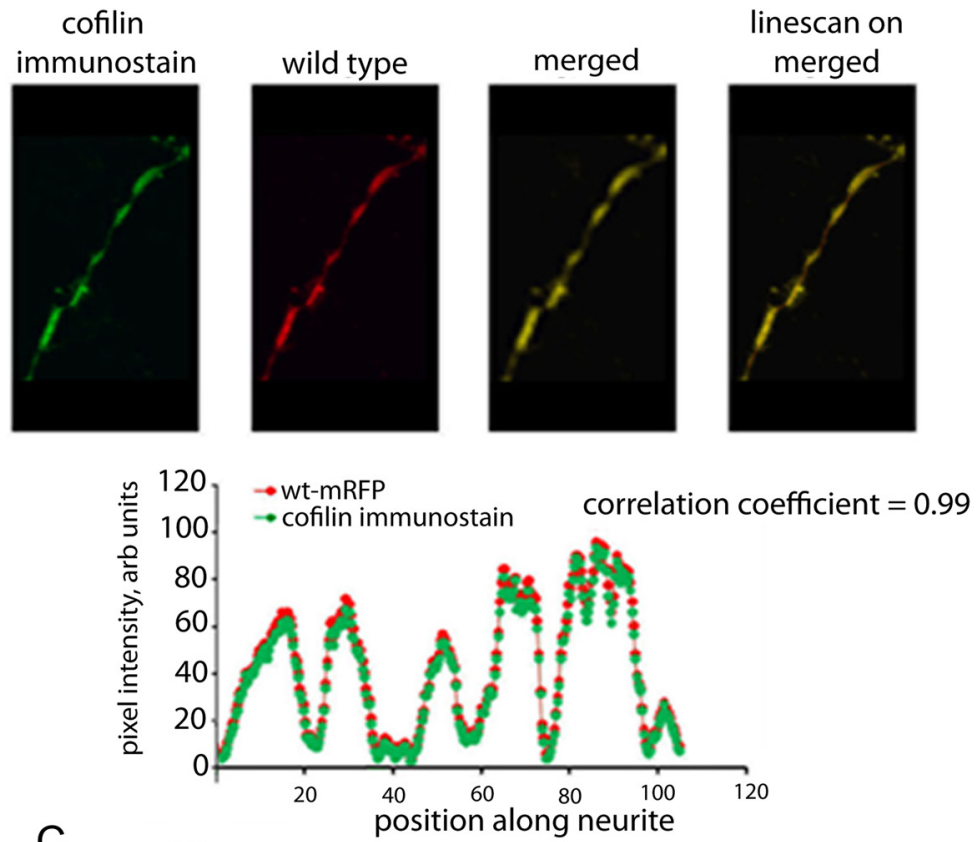

C

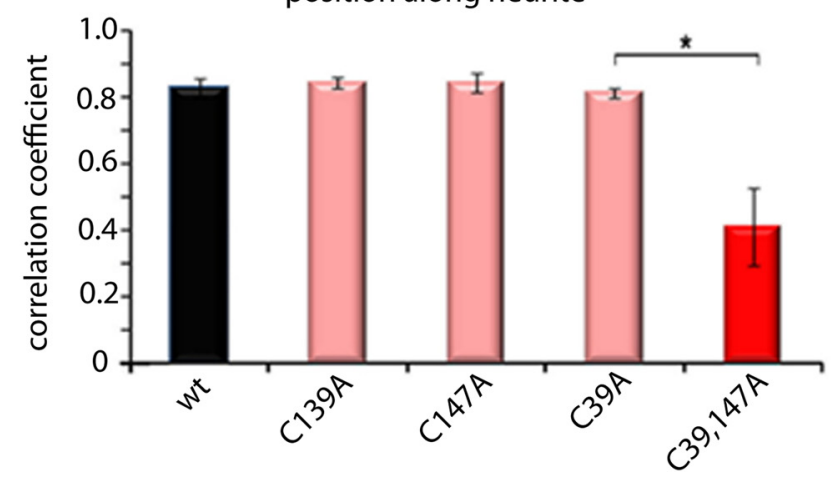

Figure 2. Of the C/A mutants tested, only CC39,147AA-mRFP, the sole mutant incapable of intermolecular bond formation, ineffectively incorporates into rods. $\boldsymbol{A}, \boldsymbol{B}$, Examples of images and line scans used to quantify protein distributions. In $\boldsymbol{A}$ the immunostained protein distribution along the length of the neurite differs visibly from that of CC39,147AA-mRFP in the images and in the intensity plots. In contrast in row $(\boldsymbol{B})$, immunostained cofilin and expressed wt-mRFP have visually indistinguishable distributions. Correlation coefficient analysis of the cofilin immunostain and cofilin-mRFP intensities in a line scan along the length of a neurite was used to compare quantitatively the distributions of stained and expressed proteins; the line scans included at least and robustly show the near absence of CC39,147AA-mRFP in cofilin immunostained rods of glutamate stressed cells; rods were detected with an antibody specific for ADF/cofilin (Shaw et al., 2004). In stark contrast to the CC39,147AA-mRFP, wt-mRFP and all single $\mathrm{C} / \mathrm{A}$ mutant cofilin-mRFP consistently incorporate into rods immunostained for cofilin. Only $25 \%$ of cells with expressed CC39,147AAmRFP and immunostained rods have CC39,147AA-mRFP in rods. In those cells with CC39,147AA-mRFP in rods, only $20 \%$ of the immunostained rods have CC39,147AA-mRFP. Thus CC39,147AA is found in $95 \%$ fewer immunostained rods than wt-mRFP or singly mutated cofilin-mRFP. Moreover, in neurites with immunostained rods showing the presence of CC39,147AA-mRFP, the distribution of immunostained cofilin and CC39,147AA-mRFP is quite different, whereas the distribution of immunostained cofilin and mRFP-tagged cofilin wt or singly mutated cofilin is similar (Fig. $1 B-E)$ as quantified below. Molecular models (Fig. $1 F$ ) show the most likely residues involved in cofilin intramolecular and intermolecular bridging (Klamt et al., 2009; Klemke et al., 2008; Pfannstiel et al., 2001) (for review, see Bernstein and Bamburg, 2010).

To compare quantitatively the distribution pattern of each exogenous protein with immunostained cofilin in neurites with rods, in the immunostained image, a one-pixel wide line was drawn through the center of the neurite along a length that included at least one rod; the line scan was then transferred from the immunostained image to the mRFP image. Correlation coefficients of the distributions of the exogenous proteins and immunostained proteins were calculated from the intensities of the pair of line scans. This analytical approach is shown in the indi-

$\leftarrow$

one immunostained rod. The plots in $\boldsymbol{A}$ and $\boldsymbol{B}$ show the protein intensities along the lines drawn through neurites in the merged images of each row. The higher the correlation coefficient the greater is the tendency of expressed protein to localize with immunostained rod protein. A perfect coincidence of localizations would have a correlation coefficient of 1.0. C, A correlation coefficient analysis of all rods in the first 18 randomly selected microscope fields of two experiments shows that all three single mutant cofilin-mRFPs incorporate into stained rods as effectively as wt-mRFP. In contrast, CC39,149AA-mRFP has a greatly reduced colocalization with stained rod cofilin $(p<0.01)$. Data are mean of two similar experiments; error bars are SEM of $>80$ rods; $(C 39,147 \mathrm{AA}$ mRFP is significantly different from other four $(p<0.01)$. Scale bar, $5 \mu \mathrm{m}$. 
vidual examples of Figure 2, $A$ and $B$, and demonstrates that mRFP-proteins with mutation of the single C39, C139, or C147 residue have the same tendency to be incorporated with high efficiency into rods as wt-mRFP (Fig. 2C). Each of these four proteins has a high correlation coefficient with immunostained cofilin. It is important to note that the single mutants can still form intermolecular C39-C147 disulfide bonds with endogenous cofilin, e.g., the C147A cofilin can still link through its unmutated C39 to the C147 of endogenous cofilin; similarly, C39A cofilin can still link through its unmutated C147 to the C39 of endogenous cofilin. The C139 can, under oxidizing conditions, engage in intramolecular disulfide bonding (Klamt et al., 2009; Klemke et al., 2008), while the C147 is involved in both intramolecular and intermolecular bonds (Klamt et al., 2009; Klemke et al., 2008; Pfannstiel et al., 2001). A significant decrease in incorporation into rods, reflected in the reduced correlation coefficient with stained cofilin, is seen only with the double mutant, CC39, 147AA (Fig. 2A,C). Hence the ability to form the C39-C147 intermolecular bond is needed for efficient incorporation into rods. We have observed similar results in human epithelial carcinoma cells line (A431) and porcine kidney cells (A4.8; data not shown). In these cases, ATP depletion, rather than glutamate excitotoxicity, was used to generate rods. The C39A, C139A, and C147A cofilin mutants fused with $\mathrm{mRFP}$ are incorporated into rods as efficiently as wt-mRFP, but rod incorporation of CC39,147AA-mRFP is greatly reduced.

The rabbit polyclonal antibody 1439 (Shaw et al., 2004), used to localize endogenous cofilin, also cross reacts with cofilinmRFPs, i.e., the endogenous form of cofilin is not the only one immunostained. This cross reactivity with cofilinmRFPs has the potential to increase erroneously the correlation coefficient between endogenous cofilin and all mRFPproteins, including CC39,147AA-mRFP. Thus, we repeated the experiments of Figures 1 and 2, but rather than comparing mRFPproteins to immunostained cofilin, we compared them to a form of wt cofilin fused to Green Fluorescent Protein (Xenopus ADF/cofilinGFP). The correlation coefficients between cofilin-mRFP proteins and XAC-GFP (rather than immunostained cofilin) also support the thesis that intermolecular disulfide bonds are needed for cofilin to be incorporated into rods (Fig. 3). Once again, CC39,147AA-mRFP only weakly colocalizes with a wt cofilin, this one GFP-tagged XAC; whereas wt-mRFP strongly colocalizes with XAC-GFP (Fig. 3E). The double mutant, with inability to form intermolecular bonds, has reduced robustness of incorporation into rods.

It is important to determine whether the double mutant interacts normally with actin, because, if it does not, it would not be expected to incorporate into rods, and thus its failure to do so would not be evidence for the importance of intermolecular cofilin bridging in rod formation. Figure 4 shows the results of equilibrium binding sedimentation studies. The CC39,147AA cofilin binds F-actin (A) and increases the supernatant actin pool (B) identically as wt cofilin. R21Q cofilin is another mutant that does not incorporate into rods, but, unlike CC39,147AA cofilin, it binds F-actin inefficiently.

The dependence of rod formation on intermolecular disulfide bonds is not unique to glutamate excitotoxic stress. Correlation coefficient analysis of cofilin immunostaining and mRFP-tagged cofilin shows the same dependence on intermolecular bonds when neurons are ATP depleted (Fig. 5A). However, ATP depletion for $30 \mathrm{~min}$ generates rods in almost three times more cells than does glutamate (Minamide et al., 2000).

The partial incorporation of CC39,147AA-mRFP into rods may simply be due to its binding to F-actin in rods that are initiated by endogenous cofilin and actin. To probe this possibility, 
A

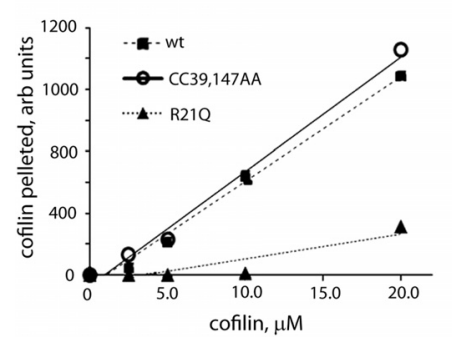

B

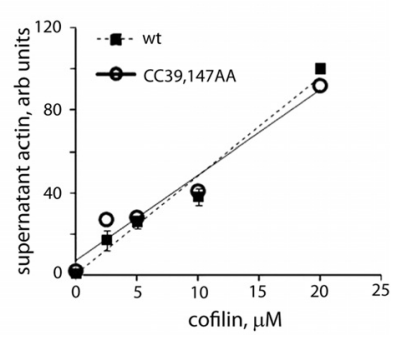

Figure 4. CC39,147AA cofilin in sedimentation assays with F-actin. Chicken muscle F-actin $(5 \mu \mathrm{M})$ was incubated with increasing concentrations of cofilin for $30 \mathrm{~min}$ before centrifugation. Cofilin in the pellet $(\boldsymbol{A})$ and actin in the supernatant $(\boldsymbol{B})$ were quantified on SDS acrylamide gels. Data were fitted with a linear trendline and show that the binding of CC39,147AA cofilin to F-actin and the increased supernatant actin are not significantly different from wt; in contrast, R21Q cofilin, another mutant with reduced rod-forming capacity, has greatly diminished F-actin binding.
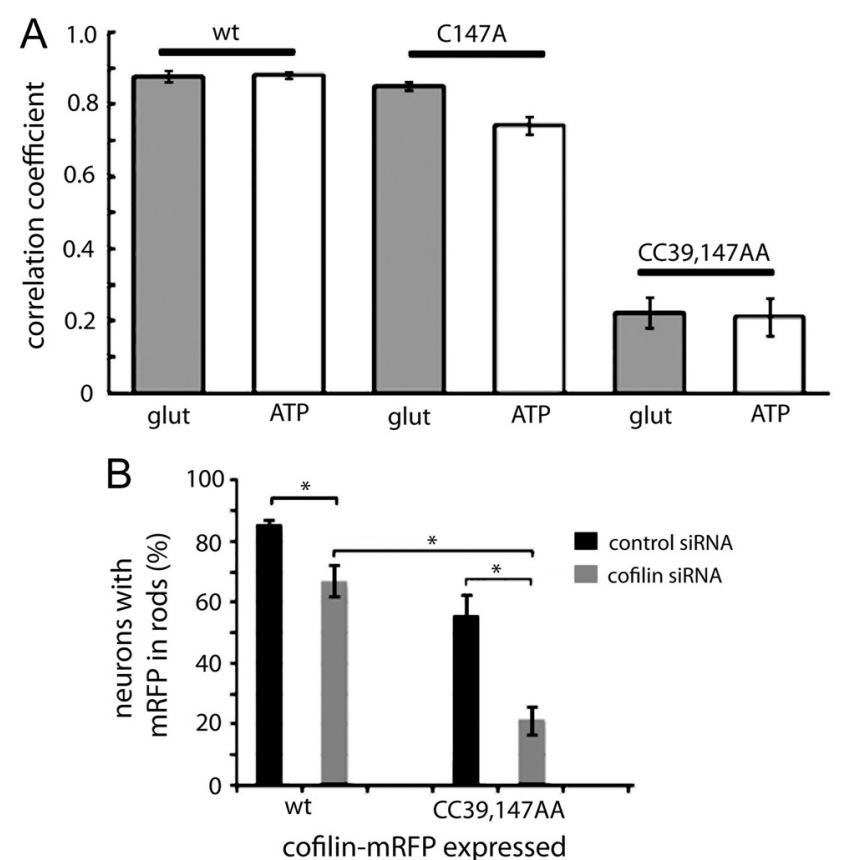

Figure 5. In cofilin-silenced hippocampal neurons, overexpressed C(39,147AA-mRFP produces fewer cells with rods than does wt-mRFP. $\boldsymbol{A}$, Experiments described in Figure 1 were repeated with either glutamate stress or ATP depletion (Minamide et al., 2000). Rods generated through energetic stress have the same dependence on cofilin intermolecular bonding as glutamate-induced rods. $\boldsymbol{B}$, Immunostaining indicated that silencing reduced cofilin 50\%. Two forms of cofilin were overexpressed, and ATP was depleted. CC39,147AA-mRFP is found in rods in far fewer cofilin-silenced cells than is wt cofilin-mRFP. Cells with $50 \%$ endogenous cofilin (i.e., those silenced for cofilin expression) incorporate less mRFP-tagged cofilin than do control cells. Data are the mean and SD of three experiments. Asterisks indicate differences significant at $p<0.01$.

rod formation from endogenous proteins was reduced by silencing cofilin. Three days after plating, hippocampal neurons were infected with an AdTrack adenovirus coexpressing behind separate promoters GFP and a hairpin RNA to generate a rat cofilin siRNA. Control hippocampal cells were infected with virus for coexpression of GFP and a hairpin RNA that has no target in rat neurons. Immunostaining of GFP-expressing cells indicated that endogenous cofilin was reduced $\sim 50 \%$ in cofilin-silenced cells compared with control cells. In control cells expressing wtmRFP, the percentage of cells with cofilin-mRFP rods $(85 \%)$ is higher than in cofilin-silenced cells (65\%). If CC39,147AA-

mRFP is expressed, rather than wt-mRFP, the percentages are further reduced to $55 \%$ for control cells and $22 \%$ for cofilinsilenced cells (Fig. $5 B$ ). Thus, in cells expressing either the double mutant or wt cofilin, the percentage of cells with cofilin-mRFP rods is lower when endogenous cofilin is lower. This supports the idea that the CC39,147AA mutant cofilin does not initiate rod formation but binds to rods formed from endogenous proteins (Fig. 4). Its inability to form intermolecular bonds reduces the robustness of its incorporation into rods, as seen in the reduced percentage of neurons with CC39,147AA-mRFP in rods compared with immunostained neurons (25\%), the reduced number of double mutant rods per neuron compared with immunostained neurons $(20 \%)$, and the low correlation coefficient for CC39,147AA-mRFP and immunostained cofilin in those few neurites that have rods (Figs. 1-3). The binding of the double mutant to actin is probably responsible for its appearance in a limited number of rods (Fig. $5 B$ ).

\section{Oxidation and rods induced by glutamate and AMPA}

If we are correct in thinking that excitotoxic levels of glutamate induce cofilin oxidation and that the oxidation of cysteine residues in cofilin is important in the formation of rods, one would expect that reducing the oxidative state of cells would decrease rods. In fact, a reducing agent (Sakatani et al., 2008) does do this when rods are generated through the overexpression of cofilinmRFP. A15 min incubation in $4 \mu \mathrm{M} \beta$-mercaptoethanol effectively reverses rods in cells at all levels of cofilin-mRFP expression (Fig. 6A,B). Parallel cultures, not exposed to reducing agent, have abundant prominent rods (Fig. $6 A$ ). Since rod formation increases with expression level, it is important to show that control and experimental cells had comparable levels of cofilinmRFP (Fig. $6 B$ ). In fact, in the duplicate experiments performed, the distribution of expression intensity in control and experimental cells was very similar (Fig. 6C).

The oxidation levels seen in and near regions of rods are consistent with oxidation being an important factor in rod formation. Thiol Tracker Violet (TTV), a fluorescent reagent, binds irreversibly to only reduced (i.e., free) sulfhydryl groups so the greater the staining intensity, the greater the reduced state (Mandavilli and Janes, 2010). In all cells, those with rods generated only through cofilin overexpression (control cells) and those cells overexpressing cofilin but also stressed by peroxide or glutamate, the rods show lower levels of free sulfhydryl groups (i.e., greater oxidation) than comparably dense regions of cofilin that lack rod morphology (Fig. 6D,E). The oxidation state of both rods and cofilin-enriched nonrod regions are similarly high in glutamateand peroxide-treated cells and exceed those of control cells (Fig. $6 E)$. This lower oxidation state in control cells (i.e., those with rods generated only by cofilin overexpression) may explain why these rods can be reversed by $\beta$-mercaptoethanol, but glutamateand peroxide-induced rods cannot (Fig. $6 A, B$ ).

We also considered the thiol staining in domains just outside rods and nonrod regions. With glutamate treatment and cofilin overexpression (control), the ratio of inside/outside TTV intensity is similar for rods and nonrod regions (Fig. $6 \mathrm{~F}$ ), indicating that the regions outside glutamate- and overexpression-induced rods also have fewer free sulfhydryl groups than nonrod regions in the same neurites. This negative correlation between sulfhydryl groups and rods suggests that oxidative state, as well as reduced ATP level (Bernstein et al., 2006), is a key factor determining the location of rod appearance in neurites.

Although TTV quantification does not indicate a lower oxidation level in glutamate-treated neurites compared with perox- 
ide treated (Fig. 6E), the greater oxidizing potency of peroxide is suggested by the greater translocation of wt cofilin to mitochondria in peroxide-treated cells compared with glutamate-treated cells (Fig. $7 A$ ). Here, correlation coefficient analysis was used to analyze the localization of different cofilin-mRFP mutants in regions of mitochondria. The fixable dye MitoTracker Green FM was used to stain all mitochondria. Peroxide drives more C139A-mRFP, as well as wt cofilin, to mitochondria, than glutamate does. Because the single C139A and single C147A mutants are each capable of forming only one of the two intramolecular disulfide bonds most likely responsible for mitochondrial translocation (Klamt et al., 2009), C139A-mRFP has less tendency to move into mitochondrial regions than does wt cofilin. Not surprisingly, mutation of both C39 and C147 eliminates the movement entirely by blocking formation of both predominant intramolecular disulfide bonds, C39-C80 and C139-C147 (Fig. 1 F).

We explored several possible mechanisms through which excitotoxic levels of glutamate may lead to cofilin oxidation in neurons. The first is by attenuating uptake of cystine. Cystine, the disulfide form of cysteine, is a rate-limiting component of glutathione synthesis, particularly important at times of stress. Glutamate excess lowers cellular glutathione by blocking cystine uptake (Schubert and Piasecki, 2001). Figure $7 B$ shows that the inclusion of $100 \mu \mathrm{M}$ cystine along with glutamate dramatically reduces the percentage of total cells with rods and the number of rods per cell (rod indices). Because the typical growth medium used has B27 supplement, which contains glutathione, it is necessary to remove the supplement to preserve cystine during the glutamate exposure. Neither this medium modification nor $30 \mathrm{~min}$ exposure to cystine alone produces rods. The second mechanism through which excitotoxic levels of glutamate can cause cofilin oxidation involves the potential energetic stress of overloading the cells with $\mathrm{Na}^{+}$and $\mathrm{Ca}^{2+}$. The elevated ATPase activity engaged to restore normal ionic gradients may deplete enough ATP to elevate reactive oxygen species (Vanden Hoek et al., 1998; Baker, 2004). Indeed, we found that ouabain, a specific inhibitor of the $\mathrm{Na}^{+} / \mathrm{K}^{+}$-ATPase, if included with glutamate during rod generation, lowers rod generation (Fig. 7C).

To further characterize glutamate rod induction, we considered the pharmacologically distinguishable glutamate receptors and found that the AMPA receptor is singularly important (Fig. $8 A$ ). Of all glutamate receptor agents tested, only AMPA and
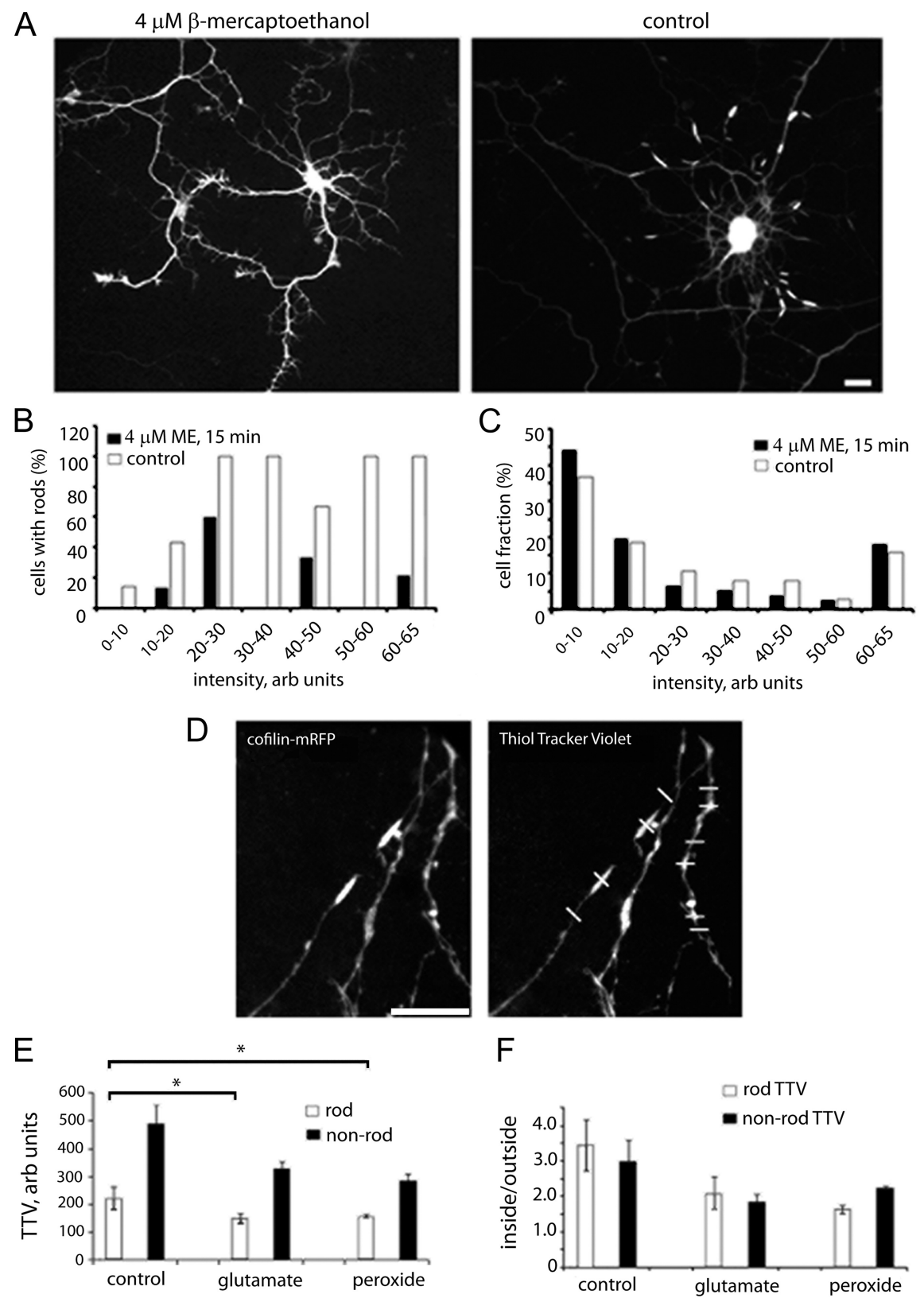

F

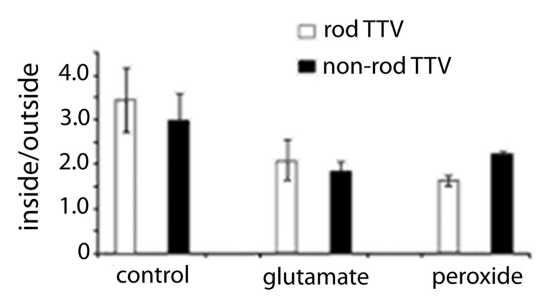

Figure 6. Measurement and manipulation of sulfhydryl oxidation in and near regions of rods. $\boldsymbol{A}, \boldsymbol{\beta}$-Mercaptoethanol reverses rods generated through wt cofilin-mRFP overexpression. These two confocal images show neurons with overexpressed wt-mRFP that were incubated for $15 \mathrm{~min}$ with and without $4 \mu \mathrm{m} \beta$-mercaptoethanol $\left(37^{\circ} \mathrm{C}\right)$ before being fixed. Images were taken of the first 20 contiguous microscope fields encountered in each of two experiments. $\boldsymbol{B}$, Because rod generation increases with the level of cofilin expressed (Minamide et al., 2000), we compared rod statistics only in cells with comparable levels of expressed wt cofilin-mRFP, hence the binning of mRFP intensity levels. C shows similar intensity distribution in control and experimental dishes measured after fixation. $\boldsymbol{D}$, Left-hand confocal image shows neurites with comparable intensities of wt cofilin-mRFP in rod and nonrod regions. Right panel shows the same microscope field, stained with Thiol Tracker Violet (TTV). TTV is a fluorescent indicator that binds free (i.e., reduced) sulfhydryl groups (Mandavilli and Janes, 2010). $\boldsymbol{E}$ shows the mean maximum TTV intensity of each line scan drawn perpendicular to the neurite through the center of the rod or nonrod regions. In control cells rods were generated through cofilin overexpression alone. In all cells TTV intensity shows higher levels of free sulfhydryls in nonrod than rod regions (E). There are also higher levels of free sulfhydryls in the rod and nonrod regions of control cells compared with those structures in glutamate- and peroxide-treated cells. Asterisk indicates significant difference at the $p<0.01$ confidence level. Rod and nonrod TTV intensity was also determined for regions adjacent to rods and nonrod regions ( $\boldsymbol{F}$ ). Data are the mean and SEM of all values from three experiments; $n \geq 150$ for each treatment. Scale bar, $10 \mu \mathrm{m}$ (in all cases).

DNQX, an AMPA antagonist, affect rod formation. DNQX, in the presence of the antagonists of NMDA and metabotropic glutamate receptors ("ant combo"), is no more effective in inhibiting glutamate induction of rods than DNQX alone. NMDA and 

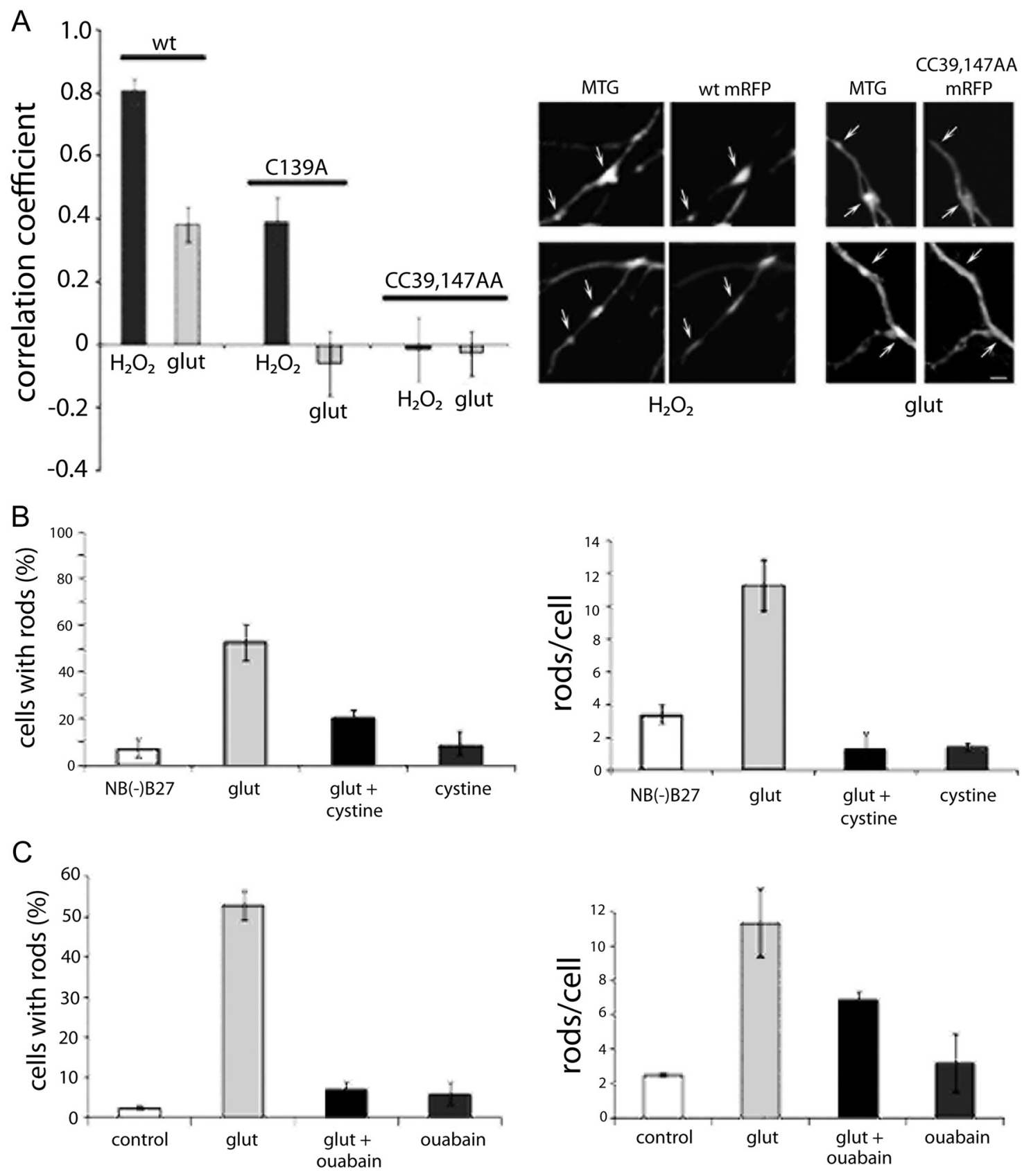

Figure 7. Aspects of glutamate-induced oxidation of cofilin: mitochondrial targeting and rod inhibition by cystine and ouabain. $A$, Hippocampal cells, expressing three different cofilin-mRFP proteins, were loaded with the fluorescent mitochondrial marker MitoTracker Green FM (MTG), stressed with either glutamate or peroxide, fixed, and imaged. Line scans along neurites and centered on mitochondria were used to measure the correlation coefficient of MitoTracker Green FM and cofilin. Arrows in confocal images indicate concentrations of MTG and either corresponding concentration of wt-mRFP or the corresponding absence of CC39,147AA-mRFP. Scale bar, $2 \mu \mathrm{m}$. B, C, Inclusion of $100 \mu \mathrm{m}$ cystine or $50 \mu \mathrm{m}$ ouabain in glutamate-stressing buffer attenuates appearance of wt-mRFP rods. In all panels data are the mean and SEM of all values from two experiments; $n \geq 90$.

the metabotropic glutamate agonist DHPG, applied together or individually, do not stimulate rod production. Moreover, AMPA stimulation is unaffected by the NMDA antagonist AP5. AMPA-rod induction shows the same dependence on in termolecular disulfide bond formation as glutamate (compare Figs. $2 C, 8 B$ ). In contrast to glutamate rod induction, rod indices with AMPA are not affected by ouabain. However, the robustness of the AMPA response is attenuated by ouabain. In ouabain-treated cells, we see a $50 \%$ decrease in wt-mRFP intensity of rods when normalized to the cell body (Fig. 8C). Presumably, ouabain preserves ATP and thus reduces oxidation (Vanden Hoek et al., 1998; Baker, 2004) necessary for cofilin-actin rod formation. Adding cystine to the AMPA/ ouabain incubation mixture had no effect on rod numbers (data not shown) probably because, unlike glutamate, AMPA does not block cystine uptake and reduce glutathione levels (Schubert and Piasecki, 2001). Our findings suggest two modes of glutamate-induced neuronal oxidative stress: ATP depletion via hyperactivation of $\mathrm{Na}^{+} / \mathrm{K}^{+}$-ATPase and glutathione depletion via blockage of cystine uptake essential for glutathione synthesis. Ouabain can attenuate the rod induction of AMPA, as well as glutamate, because AMPA and glutamate can each overload neurons with $\mathrm{Na}^{+}$, but cystine attenuates only glutamate effects. 
DTT-sensitive intermolecular cofilin bonds in rods

To test directly the importance of intermolecular disulfide bridging in rod formation in cells, we looked for SDS-stable cofilin oligomers in the rods of cortical neurons (Fig. 9A) and A431 cells, a human carcinoma epithelial cell line (Fig. 9B). Rods were isolated from ATP-depleted cells as described previously (Minamide et al., 2010) but with one important modification: cells were lysed in a buffer containing IAA, which covalently binds free sulfhydryls, thus minimizing their oxidation during the rod isolation and maximizing the chances that any oxidation product detected was formed in the intact cell. Half of the rod sample was treated with a reducing agent, DTT, before being loaded onto gels for immunoblotting; the other half was not. The higher molecular weight cofilin bands (red), those migrating $>19 \mathrm{kDa}$ (monomeric cofilin) in the absence of reducing agent, disappear when treated with DTT (Fig. 9A,B). The slowest migrating cofilin species in brackets has the mobility expected for cofilin dimer with no intramolecular bonds. Those below it are probably dimer with one or two intramolecular bonds. The yellow band (Fig. 9A, middle lane), containing actin and cofilin in species too large to exit the stacking gel, is also DTT sensitive. In contrast to isolated rods, lysates of whole cortical neurons similarly prepared and analyzed show no multimeric cofilin bands (data not shown). This is not a surprising result since, based on intensity of immunostain fluorescence, rods constitute at most $4 \%$ of the total cofilin. The absence of detectable multimeric cofilin in lysates does underscore the significance of cofilin intermolecular bonding in rod formation.

\section{Discussion}

This study suggests a new mode of cofilin regulation in cells and a mechanistic link between environmental factors and the cytoskeletal pathology of several neurodegenerative diseases. Here, we provide evidence that the cofilin intermolecular disulfide bond (C39-C147), shown to form in vitro by cofilin oxidation (Pfannstiel et al., 2001), is critical for cofilinactin rod formation in neurons stressed by glutamate, AMPA, or ATP depletion. We have also found the following: (1) that in neurites reduced sulfhydryl groups are rarer in and near regions of rods compared with nonrod compartments with similarly high cofilin concentration; (2) that rods, induced by cofilin overexpression in cells, have lower levels of oxidation than rods induced by stress and can be reversed by a disulfide reducing agent; (3) that extracellular cystine or ouabain inhibits glutamate rod formation; (4) that NMDA and a metabotropic glutamate receptor agonist do not induce rods, but the glutamate receptor agonist, AMPA, does; (5) similarly, an AMPA
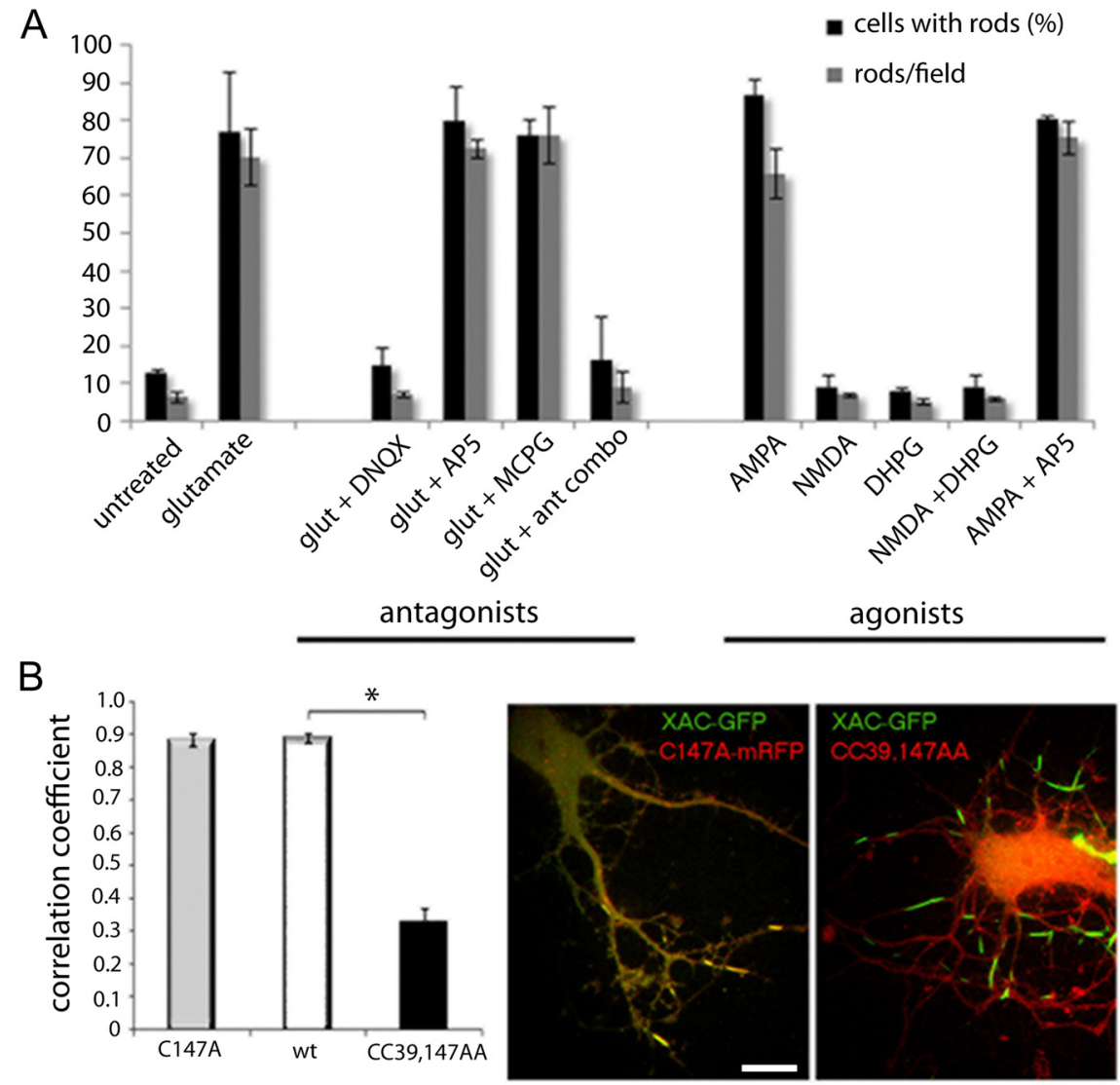

Figure 8. Within the glutamate receptor family, only activation of the AMPA receptor generates rods. $A, A M P A$, an agonist for quisqualate- or AMPA-type glutamate receptors induces rods as effectively as glutamate itself in contrast to NMDA and a metabotropic glutamate receptor agonist (DHPG). Similarly, only an AMPA receptor antagonist, DNQX, blocks rods in glutamate stressed cells. Moreover, all three glutamate antagonists combined (ant combo) are no more effective in blocking glutamate rod induction than DNQX alone. NMDA and metabotropic glutamate receptor antagonists have no effect. $\boldsymbol{B}$, With AMPA, as with glutamate (Figs. 1-3), CC39,147AA-mRFP is ineffectively incorporated into rods. Data of two experiments with $50-60$ rods/experiment. Scale bar, $10 \mu \mathrm{m}$. C, Ouabain addition to AMPA reduces wt-mRFP intensity in rods relative to somata. Data represents two experiments with $50-60$ rods/experiments. Scale bar, $20 \mu \mathrm{m}$.

receptor antagonist is able to block glutamate rod induction while NMDA and metabotropic receptor antagonists fail; (6) that cofilin movement into AMPA-induced rods depends on $\mathrm{Na}^{+} / \mathrm{K}^{+}$-ATPase, but indices of AMPA-induced rods do not; and (7) rods isolated from neurons and human epithelial cells have DTT-sensitive cofilin oligomers, notably dimers.

In 2001, Pfannstiel et al. demonstrated that cofilin in vitro can be cross-linked by the zero-distance cross-linker, Ellman's reagent (5,5'-dithio-2-nitrobenzoic acid) or by oxidized glutathione, a far milder, more physiological oxidizing reagent (Pfannstiel et al., 2001). The cofilin dimers and oligomers, so formed, no longer have the actin dynamizing activity of monomers, but do have the ability to bundle dramatically F-actin. 


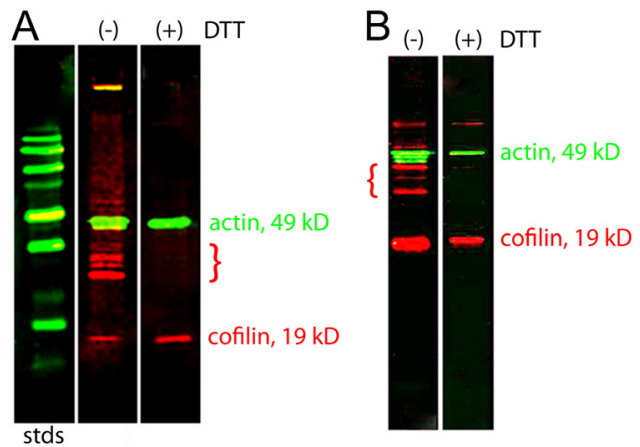

Figure 9. Rods isolated from stressed cells have DTT-sensitive higher molecular weight forms of cofilin. Rods were induced by ATP depletion of cortical cells $(\boldsymbol{A})$ or human epithelial carcinoma A431 cells $(\boldsymbol{B})$ and were isolated in the presence of IAA. Western blots for cofilin and actin show DTT-sensitive cofilin bands running at higher molecular weights than monomeric cofilin. The highest cofilin band in brackets migrates with the expected mobility of cofilin dimer with no intramolecular bonds. Lower bands in brackets are probably dimers with one or two intramolecular disulfide bonds.

Herein we focus on whether these oligomers are also involved in the formation of rods in cells when they are stressed. The ultrastructure of actin bundles formed in vitro resembles that of rods seen in nerve cells (Maloney et al., 2005; Minamide et al., 2000). Moreover, most stresses, capable of generating rods in cells, also are immediately or eventually oxidizing. HPLC analysis of cofilin proteolytic fragments revealed that mild oxidative stress is likely to cause disulfide bonding between C39 and C147 of neighboring cofilin molecules (Pfannstiel et al., 2001). More severe stress also involves $\mathrm{C} 80$ and $\mathrm{C} 139$, generating one or even two intramolecular disulfide bonds that distort the molecular structure. Cofilin dimers and cofilin with a single intramolecular bond can still bind actin, but not depolymerize it (Klemke et al., 2008; Pfannstiel et al., 2001). Two intramolecular bonds distort cofilin enough to prevent actin binding and produce a novel function, the initiation of apoptosis. Cofilin with two intramolecular bonds opens the permeability transition pore of mitochondria, releases cytochrome $c$ (Luetjens et al., 2000), and thus triggers apoptosis (Klamt et al., 2009).

To elucidate the link between cofilin oxidation and the cytoskeletal pathologies of familial and sporadic $\mathrm{AD}$, we chose glutamate as a model of oxidizing stress. Almost all rod-inducing stress produces extracellular accumulation of glutamate, and excitotoxic levels of glutamate elevate reactive oxygen species (Reynolds and Hastings, 1995). Rods were found to be more abundant in $\mathrm{AD}$ brain than in brain of cognitively normal individuals (Minamide et al., 2000). They appear to be part of a feedforward mechanism of $\mathrm{AD}$ that has the potential to explain the accelerating cognitive decline typical of the disease. The amyloid- $\beta$ peptide induces rods in the dentate gyrus (Davis et al., 2009, 2011), a structure with known learning and memory importance, and also potentiates secretion of amyloid- $\beta$ (Marsden et al., 2011). Moreover, rods appear to provide a scaffold for the accumulation of phosphorylated tau which may enhance its conversion to neuropil threads. These threads are a hallmark of AD whose abundance correlates with the disease severity (Giannakopoulos et al., 2007). If cofilin is silenced, phosphorylated tau is not recruited into stress-induced striations (Whiteman et al., 2009; Bamburg et al., 2010), the likely forerunner of striated neuropil threads (Velasco et al., 1998). Hence, oxidative stress and rods likely could mediate the transition of normal brain into sporadic $\mathrm{AD}$ brain. Interestingly, protein oxidation, rather than lipid oxidation, is thought to be a key factor in the progression of mild cognitive impairment to fully blown AD (Baldeiras et al., 2010).

Glutamate and peroxide differ in their oxidizing effects on cofilin: peroxide generates rods in $80 \%$ fewer cells than glutamate, causes cell death $24 \mathrm{~h}$ after washout (Minamide et al., 2000), and, as shown herein, is much more effective in targeting cofilin to mitochondria. The single mutant, C139A, can incorporate into rods as efficiently as wt cofilin, but it moves to mitochondria much less efficiently than wt. This reduction is consistent with two intramolecular bonds being needed for cofilin-dependent apoptosis (Klamt et al., 2009) and extends that finding to primary neurons. These results are also consistent with cofilin C39-C147 intermolecular bond formation being associated with rod formation and not mitochondrial translocation. The double mutant is our only tested species incapable of intermolecular bond formation, and the only species that has robustly reduced incorporation into rods. The simplest explanation for CC39,147AA moving to mitochondria even less effectively than C139 is the inability of CC 39,147AA to form any intramolecular (or intermolecular) disulfide bonds.

Glutamate can modulate cofilin activity through effects on ATP levels, some of which are calcium related. Extreme levels of intracellular calcium can deplete ATP either by over working ATPases, meant to maintain homeostatic ion levels, or by impairing mitochondrial oxidative phosphorylation. ATP depletion is of particular concern with regard to rod formation. First, rapid cofilin dephosphorylation and generation of reactive oxygen species follow ATP depletion. Second, chronophin, a specific cofilin phosphatase, unrelated to slingshot, is activated. Chronophin is a member of the haloacid dehalogenase (HAD) superfamily (Gohla et al., 2005) whose inhibition by hsp90 binding is ATP dependent. Hence a decline in ATP results in chronophin activation of cofilin that was shown to be necessary for rod formation in HeLa cells and primary neurons (Huang et al., 2008).

The oxidative effects of glutamate may stem also from either reactive oxygen species associated with mitochondrial damage or inhibition of cystine uptake (Schubert and Piasecki, 2001). Inhibition of cystine uptake and a drop in ATP exhaust reserves of the cell's main reducing agent, glutathione. Glutathione synthesis depends on cystine uptake and ATP. The ensuing oxidative stress could activate cofilin and promote rods by releasing slingshot from an activity-inhibiting complex with the multifunctional sig-

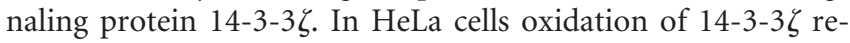
leases cofilin and generates rods (Kim et al., 2009). In those HeLa cells, which stably express eGFP-cofilin $1,500 \mu \mathrm{M}$ peroxide does not oxidize cofilin, whereas in human T cells as little as $50 \mu \mathrm{M}$ peroxide does. HeLa cells may deactivate reactive oxygen species more effectively than human $\mathrm{T}$ cells.

Glutamate induction of rods undoubtedly involves multiple receptors and pathways, only some of which include calcium transients. The phosphorylated, inactive form of cofilin does not bind actin and does not incorporate into rods. Overexpression of LIM kinase is sufficient to prevent neuronal rod formation induced by amyloid- $\beta$ (Davis et al., 2009). Cofilin dephosphorylation, which precedes rod formation, is likely in part a consequence of calcium influx and the integration of multiple, interlacing calcium-dependent pathways (Carlisle et al., 2008). When conditions favor rod formation, the integration of these pathways is expected to cause net dephosphorylation and consequently abnormally high levels of active cofilin (Davis et al., 2011). The calcium-dependent pathways include the following: (1) calcineurin dephosphorylating and activating slingshot (Wang et al., 2005) that activates cofilin directly by dephospho- 
rylating it or indirectly by dephosphorylating LIMK (Soosairajah et al., 2005); and (2) pCaMKII, p-synGAP, RasGRF1, PI3 kinase, Tiam1, and Rac that are upstream of PAK and can inactivate cofilin via LIMK or slingshot. We think the kinetics and/or balance of intensity of these glutamate-responsive, rod-forming pathways could be shifted to produce instead preconditioning, long-term depression, long-term potentiation, or lethality.

Yet another factor contributing to the probability of glutamate-inducing rods is $\mathrm{pH}$ modulation of cofilin activity (Bernstein et al., 2000; Frantz et al., 2008; Pavlov et al., 2006; Zhao et al., 2010), since the energetic and oxidative stress of excitotoxic glutamate acidifies cells (Reynolds and Hastings, 1995). Acidification potentiates the salt bridges that promote the inactivation of cofilin through binding to phosphatidyl inositol 4,5 bisphosphate (Zhao et al., 2010; Frantz et al., 2008). If acidification occurred rapidly enough, it could attenuate the interaction of cofilin and actin and inhibit rod formation.

Any process that modulates cofilin activity potentially alters the probability of rod formation. Interestingly, a single stress, such as ATP depletion, may generate a plethora of rods in minutes in one cell type and none in another. This range of cell responses probably reflects the complexity and variability of cofilin phospho-regulation and other cofilin modulatory mechanisms (Bernstein and Bamburg, 2010). A detailed study of the conditions and mechanisms that result in glutamate-inducing rods rather than LTP, preconditioning, or cell death should enhance our understanding of rod function in neuronal cell biology. From the current study of acutely dissociated, cultured neurons, we have learned that oxidation, responsible for intermolecular bonding of cofilin, is also a likely cause of synaptic loss and a potential mode of actin regulation in cells.

\section{References}

Baker JE (2004) Oxidative stress and adaptation of the infant heart to hypoxia and ischemia. Antioxid Redox Signal 6:423-429.

Baldeiras I, Santana I, Proença MT, Garrucho MH, Pascoal R, Rodrigues A, Duro D, Oliveira CR (2010) Oxidative damage and progression to Alzheimer's disease in patients with mild cognitive impairment. J Alzheimers Dis 21:1165-1177.

Bamburg JR, Bernstein BW, Davis RC, Flynn KC, Goldsbury C, Jensen JR, Maloney MT, Marsden IT, Minamide LS, Pak CW, Shaw AE, Whiteman I, Wiggan O (2010) ADF/cofilin-actin rods in neurodegenerative diseases. Curr Alzheimer Res 7:241-250.

Bernstein BW, Bamburg JR (2003) Actin-ATP hydrolysis is a major energy drain for neurons. J Neurosci 23:1-6.

Bernstein BW, Bamburg JR (2010) ADF/cofilin: a functional node in cell biology. Trends Cell Biol 20:187-195.

Bernstein BW, Painter WB, Chen H, Minamide LS, Abe H, Bamburg JR (2000) Intracellular $\mathrm{pH}$ modulation of $\mathrm{ADF} /$ cofilin proteins. Cell Motil Cytoskeleton 47:319-336.

Bernstein BW, Chen H, Boyle JA, Bamburg JR (2006) Formation of actin$\mathrm{ADF} /$ cofilin rods transiently retards decline of mitochondrial potential and ATP in stressed neurons. Am J Physiol Cell Physiol 291:C828-839.

Campbell RE, Tour O, Palmer AE, Steinbach PA, Baird GS, Zacharias DA, Tsien RY (2002) A monomeric red fluorescent protein. Proc Natl Acad Sci U S A 99:7877-7882.

Carlisle HJ, Manzerra P, Marcora E, Kennedy MB (2008) SynGAP regulates steady-state and activity-dependent phosphorylation of cofilin. J Neurosci 28:13673-13683.

Cleary JP, Walsh DM, Hofmeister JJ, Shankar GM, Kuskowski MA, Selkoe DJ, Ashe KH (2005) Natural oligomers of the amyloid-beta protein specifically disrupt cognitive function. Nat Neurosci 8:79-84.

Davis RC, Maloney MT, Minamide LS, Flynn KC, Stonebraker MA, Bamburg JR (2009) Mapping cofilin-actin rods in stressed hippocampal slices and the role of cdc42 in amyloid-beta-Induced rods. J Alzheimers Dis 18:35-50.

Davis RC, Marsden IT, Maloney MT, Minamide LS, Podlisny M, Selkoe DJ, Bamburg JR (2011) Amyloid beta dimers/trimers potently induce cofilin- actin rods that are inhibited by maintaining cofilin-phosphorylation. Mol Neurodegener 6:10.

Frantz C, Barreiro G, Dominguez L, Chen X, Eddy R, Condeelis J, Kelly MJ, Jacobson MP, Barber DL (2008) Cofilin is a $\mathrm{pH}$ sensor for actin free barbed end formation: role of phosphoinositide binding. J Cell Biol 183:865-879.

Garvalov BK, Flynn KC, Neukirchen D, Meyn L, Teusch N, Wu X, Brakebusch C, Bamburg JR, Bradke F (2007) Cdc42 regulates cofilin during the establishment of neuronal polarity. J Neurosci 27:13117-13129.

Giannakopoulos P, von Gunten A, Kövari E, Gold G, Herrmann FR, Hof PR, Bouras C (2007) Stereological analysis of neuropil threads in the hippocampal formation: relationships with Alzheimer's disease neuronal pathology and cognition. Neuropathol Appl Neurobiol 33:334-343.

Giuliano KA, Khatib FA, Hayden SM, Daoud EW, Adams ME, Amorese DA, Bernstein BW, Bamburg JR (1988) Properties of purified actin depolymerizing factor from chick brain. Biochemistry 27:8931-8938.

Gohla A, Birkenfeld J, Bokoch GM (2005) Chronophin, a novel HAD-type serine protein phosphatase, regulates cofilin-dependent actin dynamics. Nat Cell Biol 7:21-29.

Herrup K (2010) Reimagining Alzheimer's disease-an age-based hypothesis. J Neurosci 30:16755-16762.

Huang TY, Minamide LS, Bamburg JR, Bokoch GM (2008) Chronophin mediates an ATP-sensing mechanism for cofilin dephosphorylation and neuronal cofilin-actin rod formation. Dev Cell 15:691-703.

Jang DH, Han JH, Lee SH, Lee YS, Park H, Lee SH, Kim H, Kaang BK (2005) Cofilin expression induces cofilin-actin rod formation and disrupts synaptic structure and function in Aplysia synapses. Proc Natl Acad Sci U S A 102:16072-16077.

Kim JS, Huang TY, Bokoch GM (2009) Reactive oxygen species (ROS) regulate a slingshot-cofilin activation pathway. Mol Biol Cell 20:2650-2660.

Klamt F, Zdanov S, Levine RL, Pariser A, Zhang Y, Zhang B, Yu LR, Veenstra TD, Shacter E (2009) Oxidant-induced apoptosis is mediated by oxidation of the actin-regulatory protein cofilin. Nat Cell Biol 11:1241-1246.

Klemke M, Wabnitz GH, Funke F, Funk B, Kirchgessner H, Samstag Y (2008) Oxidation of cofilin mediates T cell hyporesponsiveness under oxidative stress conditions. Immunity 29:404-413.

Li S, Hong S, Shepardson NE, Walsh DM, Shankar GM, Selkoe D (2009) Soluble oligomers of amyloid Beta protein facilitate hippocampal longterm depression by disrupting neuronal glutamate uptake. Neuron 62:788-801.

Luetjens CM, Bui NT, Sengpiel B, Münstermann G, Poppe M, Krohn AJ, Bauerbach E, Krieglstein J, Prehn JH (2000) Delayed mitochondrial dysfunction in excitotoxic neuron death: cytochrome c release and a secondary increase in superoxide production. J Neurosci 20:5715-5723.

Maloney MT, Minamide LS, Kinley AW, Boyle JA, Bamburg JR (2005) Betasecretase-cleaved amyloid precursor protein accumulates at actin inclusions induced in neurons by stress or amyloid beta: a feedforward mechanism for Alzheimer's disease. J Neurosci 25:11313-11321.

Mandavilli BS, Janes MS (2010) Detection of intracellular glutathione using ThiolTracker violet stain and fluorescence microscopy. Curr Protoc Cytom Chap 9:Unit 9.35.

Marsden IT, Minamide LS, Bamburg JR (2011) Amyloid-beta-induced amyloid-beta secretion: a possible feed-forward mechanism in Alzheimer's disease. J Alzheimers Dis 24:681-691.

Mc Donald JM, Savva GM, Brayne C, Welzel AT, Forster G, Shankar GM, Selkoe DJ, Ince PG, Walsh DM (2010) The presence of sodium dodecyl sulphate-stable Abeta dimers is strongly associated with Alzheimer-type dementia. Brain 133:1328-1341.

Minamide LS, Striegl AM, Boyle JA, Meberg PJ, Bamburg JR (2000) Neurodegenerative stimuli induce persistent $\mathrm{ADF} /$ cofilin-actin rods that disrupt distal neurite function. Nat Cell Biol 2:628-636.

Minamide LS, Shaw AE, Sarmiere PD, Wiggan O, Maloney MT, Bernstein BW, Sneider JM, Gonzalez JA, Bamburg JR (2003) Production and use of replication-deficient adenovirus for transgene expression in neurons. Methods Cell Biol 71:387-416.

Minamide LS, Maiti S, Boyle JA, Davis RC, Coppinger JA, Bao Y, Huang TY, Yates J, Bokoch GM, Bamburg JR (2010) Isolation and characterization of cytoplasmic cofilin-actin rods. J Biol Chem 285:5450-5460.

Ohm TG (2007) The dentate gyrus in Alzheimer's disease. Prog Brain Res 163:723-740.

Pardee JD, Spudich JA (1982) Purification of muscle actin. Methods Enzymol 85 [Pt B]:164-181. 
Pavlov D, Muhlrad A, Cooper J, Wear M, Reisler E (2006) Severing of $\mathrm{F}$-actin by yeast cofilin is $\mathrm{pH}$-independent. Cell Motil Cytoskeleton 63:533-542.

Pfannstiel J, Cyrklaff M, Habermann A, Stoeva S, Griffiths G, Shoeman R, Faulstich H (2001) Human cofilin forms oligomers exhibiting actin bundling activity. J Biol Chem 276:49476-49484.

Reynolds IJ, Hastings TG (1995) Glutamate induces the production of reactive oxygen species in cultured forebrain neurons following NMDA receptor activation. J Neurosci 15:3318-3327.

Sakatani M, Yamanaka K, Kobayashi S, Takahashi M (2008) Heat shockderived reactive oxygen species induce embryonic mortality in in vitro early stage bovine embryos. J Reprod Dev 54:496-501.

Schubert D, Piasecki D (2001) Oxidative glutamate toxicity can be a component of the excitotoxicity cascade. J Neurosci 21:7455-7462.

Shaw AE, Minamide LS, Bill CL, Funk JD, Maiti S, Bamburg JR (2004) Cross-reactivity of antibodies to actin- depolymerizing factor/cofilin family proteins and identification of the major epitope recognized by a mammalian actin-depolymerizing factor/cofilin antibody. Electrophoresis 25:2611-2620.

Soosairajah J, Maiti S, Wiggan O, Sarmiere P, Moussi N, Sarcevic B, Sampath R, Bamburg JR, Bernard O (2005) Interplay between components of a novel LIM kinase-slingshot phosphatase complex regulates cofilin. EMBO J 24:473-486.

Vanden Hoek TL, Becker LB, Shao Z, Li C, Schumacker PT (1998) Reactive oxygen species released from mitochondria during brief hypoxia induce preconditioning in cardiomyocytes. J Biol Chem 273:18092-18098.

Velasco ME, Smith MA, Siedlak SL, Nunomura A, Perry G (1998) Striation is the characteristic neuritic abnormality in Alzheimer disease. Brain Res 813:329-333.

Wang Y, Shibasaki F, Mizuno K (2005) Calcium signal-induced cofilin dephosphorylation is mediated by slingshot via calcineurin. J Biol Chem 280:12683-12689.

Wessel D, Flügge UI (1984) A method for the quantitative recovery of protein in dilute solution in the presence of detergents and lipids. Anal Biochem 138:141-143.

Whiteman IT, Gervasio OL, Cullen KM, Guillemin GJ, Jeong EV, Witting PK, Antao ST, Minamide LS, Bamburg JR, Goldsbury C (2009) Activated actindepolymerizing factor/cofilin sequesters phosphorylated microtubuleassociated protein during the assembly of alzheimer-like neuritic cytoskeletal striations. J Neurosci 29:12994-13005.

Zhao H, Hakala M, Lappalainen P (2010) ADF/cofilin binds phosphoinositides in a multivalent manner to act as a PIP(2)-density sensor. Biophys J 98:2327-2336. 